\title{
Performance stability of solid-state polypyrrole-reduced graphene oxide-modified carbon bundle fiber for supercapacitor application
}

\begin{abstract}
The stability performance of a solid-state polypyrrole-reduced graphene oxide (PPy-rGO) supercapacitor electrode after a series of charging-discharging cycles is investigated. The electrochemical performances show that the capacity retention, specific capacitance, equivalent series resistance, and charge transfer resistance all decrease after chargingdischarging cycles. Thermogravimetric analysis shows the degradation of electroactive materials, causing decreased electrochemical performance of PPy-rGO. The morphology changes reveal that the pore size is reduced by $\sim 12 \mu \mathrm{m}$ at the 1000th charge-discharge cycle, which limits the diffusion of electrolyte ions into the electrode. The positive shifts in the binding energy of the N1s spectra at the 500th and 1000th charge-discharge cycles indicate the formation of a hydrogen-bridge bond, affecting the electron transfer in the PPy-rGO composite as observed through X-ray photoelectron spectroscopy. Moreover, the structural properties of rGO change from amorphous to graphitic after a series of charging-discharging processes, as shown by the Raman spectra. Furthermore, the peak of the NH bending vibration is red-shifted by approximately $108 \mathrm{~cm}-1$, indicating the changes in the chemical environment after a series of charging-discharging cycles, as shown by Fourier transform infrared spectroscopy.
\end{abstract}

Keyword: Graphene; Polypyrrole; Supercapacitor; Stability 